\title{
Propiedades psicométricas de un instrumento de mecanismos de desconexión moral: validación en adolescentes del noroeste de México
}

\author{
Gildardo Bautista ${ }^{1}$, José Ángel Vera ${ }^{2}$, María Clara Cuevas ${ }^{3}$, y Jesús Tánori ${ }^{4}$ \\ ${ }^{1}$ Universidad Intercultural del Estado de Puebla (México); ${ }^{2}$ Centro de Investigación en \\ Alimentación y Desarrollo (México); ${ }^{3}$ Pontificia Universidad Javeriana (Colombia); \\ ${ }^{4}$ Instituto Tecnológico de Sonora (México)
}

\begin{abstract}
El objetivo del estudio fue traducir, adaptar y evaluar las evidencias de validez, por constructo y concurrente, del instrumento que mide los mecanismos de desconexión moral en adolescentes escolarizados. Se analiza la evidencia de validez concurrente con el rol de espectador-alentador de la agresión. Participaron 1,212 estudiantes de 10 secundarias públicas de cinco municipios de un Estado del noroeste de México. El 54.4\% son mujeres y el $45.6 \%$ hombres, cuyo rango de edad está comprendido entre los 11 y 15 ( $\mathrm{M}$ edad $=13.08, \mathrm{DE}=.95$ años $)$. Se obtuvo un modelo de medida que confirma con indicadores de ajuste aceptables internos y se evidencia la validez concurrente. Los resultados se discuten en términos de su utilidad para identificar el constructo en un ecosistema cultural particular y para el análisis contextualizado. Se concluye que la escala cuenta con propiedades psicométricas adecuadas para la medición de la desconexión moral en adolescentes, concretando, además, un aporte al estudio de la violencia y el acoso escolar.
\end{abstract}

Palabras clave: Desconexión moral, adolescentes, psicometría, modelo de medida, observadores.

Psychometric properties of an instrument for measuring mechanisms of moral disengagement: validation in adolescents from northwestern Mexico. The objective of the study was to translate, adapt and evaluate the evidence of construct and concurrent validity of an instrument that measures the mechanisms of moral disengagement in school adolescents. The evidence of concurrent validity with the role of spectator-encouraging of the aggression is analyzed. 1,212 students from 10 public high schools from five municipalities of a northwestern state of Mexico participated. 54.4\% are women and $45.6 \%$ are men, whose age range is between 11 and 15 ( $\mathrm{M}$ age $=13.08, \mathrm{SD}=.95$ years). $\mathrm{A}$ measurement model that confirms concurrent validity with acceptable internal fit indicators was obtained. The results are discussed in terms of their usefulness for identifying the construct in a particular cultural ecosystem and for contextualized analysis. It is concluded that the scale has adequate psychometric properties for the measurement of moral disengagement in adolescents, specifying, in addition, a contribution to the study of violence and school bullying.

Keywords: Moral disengagement, adolescents, psychometry, measurement model, bystanders.

Correspondencia: Jesús Tánori Quintana. Departamento de Educación. Instituto Tecnológico de Sonora. Calle 5 de Febrero 818, Centro, Urb. No 1. C.P.: 85000. Ciudad Obregón, Sonora (México). E-mail: jesus.tanori@itson.edu.mx 
La violencia es una de las problemáticas más importantes que enfrenta actualmente toda la sociedad, tanto que ha sido incluida como una de las metas globales de la Agenda 2030 para el Desarrollo Sostenible, donde se aboga por su disminución a través de la promoción de sociedades de paz e inclusión, del acceso a la justicia y la construcción de instituciones responsables y eficaces (Comisión Económica para América Latina y el Caribe [CEPAL], 2016).

Dentro de las múltiples manifestaciones de violencia, merecen especial atención aquellas que ocurren en los espacios que se encuentran bajo la responsabilidad y tutela de las instituciones que le son propias al Estado. Uno de estos espacios es el que corresponde a las escuelas y es un derecho, entonces, garantizar un ambiente libre de violencia dentro del contexto educativo y tener un entorno apropiado para el aprendizaje e interacción entre los adolescentes estudiantes (CEPAL, 2017), pues es en las escuelas donde se manifiestan conductas agresivas físicas, verbales, relacionales, intencionales, que se reproducen de manera sistemática entre un estudiante o un grupo de estudiantes contra otro estudiante, cuyo poder físico o social en el contexto escolar es bajo, por lo que le es difícil defenderse, lo cual ha sido denominado como bullying o acoso escolar (Cuevas y Marmolejo, 2016; Olweus, Solberg, y Breivik, 2020).

Diferentes estudios publicados recientemente ponen en evidencia que el bullying o acoso escolar es un problema global presente en todas las escuelas, de las que no son excepción las de los países latinoamericanos. Dichos estudios reportan que entre $4.6 \%$ y $50 \%$ de estudiantes son víctimas, y que entre $4 \%$ y $34.9 \%$ de estudiantes son agresores (Garaigordobil, Mollo-Torrico y Larrain, 2018; Herrera-López, Romera y Ortega-Ruiz, 2018). Según un estudio de metaanálisis, en México entre 19\% y $21 \%$ de la comunidad estudiantil se ve afectada y "uno de cada cinco estudiantes mexicanos recibe o realiza bullying o cyberbyllying” (Vega-Cauich, 2019, p. 113).

Teniendo en cuenta las implicaciones sociales que tiene el bullying o acoso escolar, este puede considerarse como un acto punitivo, ya que viola los derechos de los estudiantes, como lo son el respeto a su dignidad y a tener una educación de calidad. Ante la necesidad de encontrar las variables con las cuales está relacionado, algunos estudios (Campaert, Nocentini, y Menesini, 2017; Caravitaa, Strohmeierb, Salmivalli, y Di Blasioa, 2019; Meter y Bauman, 2018; Gini, Thornberg, y Pozzoli, 2020) han propuesto como marco de referencia explicativo a la teoría social cognitiva de la agencia moral, la cual supone que los individuos buscan establecer pautas comportamentales que les permitan percatarse de la utilidad de inhibir ciertos actos que afectan a sus semejantes y a ellos mismos en la búsqueda del bienestar individual y comunitario (Bandura, 2016).

En la teoría social cognitiva de agencia moral se sostiene que durante el proceso de socialización el individuo aprende las conductas, normas y valores morales del ecosistema en el que vive a través de experiencias directas e indirectas (Bandura, 2016; Bandura, Barbaranelli, Caprara, y Pastorelli, 1996). Es durante este proceso de 
socialización que el individuo adopta estándares morales -normas y valores-, que le sirven como guías para su comportamiento, lo que le permite desarrollar a su vez mecanismos de control para obrar de acuerdo con esos estándares, que son inhibitorios y activadores. Así, cuando el individuo inhibe conductas inmorales y, por ende, actúa moralmente, se supone que ejerce agencia moral, lo que implica su acción autónoma e intencional (Bandura, 1990).

Las normas y valores funcionan como reguladores de la conducta, en el sentido de que la persona se abstiene de realizar conductas antisociales e intenta comportarse de manera prosocial (Bandura, 2002). Comportarse de acuerdo con un sistema normativo implicaría que el sistema regulador de la conducta moral se ha puesto en marcha. Por ejemplo, el sujeto anticipa la consecuencia que conlleva la culpa si se comporta de manera moralmente incorrecta, que va desde lo mal que se sentiría consigo mismo, hasta anticipar el daño que podría causar a otras personas (Bandura, 2016; Ortega, Sánchez, y Menesini, 2002).

Sin embargo, Bandura $(2002,2016)$ menciona que el sistema regulador de la conducta moral es inestable y depende fundamentalmente del contexto específico debido a que es frecuente encontrar situaciones en las cuales los seres humanos se comportan de manera contraria a sus estándares morales. Para explicar estos comportamientos, Bandura (1975, 1990) utilizó el constructo denominado mecanismos de desconexión moral, el cual indica que las personas pueden corromper el sistema de autorregulación utilizando un conjunto de mecanismos cognitivos para auto-reforzar, disminuir y exonerarse de la culpa y malestar cuando sus acciones no corresponden con sus estándares morales. Estos mecanismos cognitivos pueden ser de ocho tipos (Bandura, 1975; Bandura, 2016; Bandura et al., 1996; Paulino, Avilés, y Sales, 2016) a saber:

(a) Comparación ventajosa: Intentar disminuir los daños de las acciones agresivas comparándolas con hechos más graves (golpear a los demás es peor que gritarle); (b) justificación moral: Justificar la conducta en función de principios más elevados considerándolo una causa digna (lo golpeo para que aprenda); (c) desplazamiento de la responsabilidad: Se busca transferir la responsabilidad de la conducta a un tercero implicado (le hice daño porque alguien me dijo); (d) difusión de la responsabilidad: Se minimiza y dispersa la responsabilidad sobre la conducta personal. No existe una responsabilidad personal (le hice daño, pero todos lo hicimos); (e) deshumanización de la víctima: Consiste en despojar a las víctimas de cualidades humanas considerándolas merecedoras de conductas violentas (le hice daño porque es odioso); (f) atribución de culpa a las víctimas: Con este mecanismo las personas se ven a sí mismas como personas de buena voluntad, pero forzadas a realizar ataques en contra de adversarios infames (lo golpeo porque se deja y nunca dice nada); (g) distorsión de las consecuencias: Consiste en tergiversar, minimizar o distorsionar el impacto de las conductas y pasar por alto los daños o restarles importancia (burlarse de los demás no 
hace daño); (h) etiquetamiento eufemístico: Consiste en denominar de manera diferente la acción, llamarla chiste, broma, estrategia de corrección, ente otras (no le hice daño, solo jugábamos).

En el modelo teórico original los ocho mecanismos de desconexión moral se agrupan a su vez en cuatros categorías de mecanismos cognitivos, los cuales son: reinterpretar la conducta inmoral, disminuir la responsabilidad personal, ignorar las consecuencias de las conductas y culpar a los que son víctimas de las conductas inmorales (Bandura, 2002; Bandura, 2016; Ortega et al., 2002).

En el caso del estudio del bullying o acoso escolar, el constructo se ha utilizado para analizar y explicar la conducta de quienes lo realizan, así como la conducta de los escolares que lo presencian sin defender a las víctimas o ayudando a los agresores, dando como resultado, de manera general, una correlación alta y positiva entre tales conductas y los mecanismos de desconexión moral. Dichas correlaciones han sido reportadas en países como Italia (Bandura, Caprara, Barbaranelli, Pastorelli, y Regalia, 2001), Estados Unidos (Pelton, Gound, Forehand, y Brody, 2004; Wang, Hoon, Swearer, Turner, y Goldberg, 2017), Dinamarca (Obermann, 2011a, 2011b), México (Haro, 2014; López y Kyriacou, 2016), Suiza (Bjärehed, Thornberg, Wänström, y Gini, 2019), España (Ortega et al., 2002), Noruega (Thornberg y Jungert, 2013), entre otros.

Para medir el constructo mecanismos de desconexión moral, Bandura et al. (1996) desarrollaron un instrumento con 32 reactivos distribuidos teóricamente en las ocho dimensiones de desconexión moral. Dicho instrumento ha sido objeto de varios procesos de validación y adaptación, entre ellos el realizado por Obermann (2011b) con un grupo de 667 niños y adolescentes de escuelas primarias danesas. Asimismo, con la escala original de Bandura et al. (1996), Pelton et al. (2004) realizaron un análisis con componentes principales y rotación varimax con 245 niños afroamericanos del suroeste de Estados Unidos, lo que resultó en la supresión de los cuatro reactivos que corresponden a la dimensión de lenguaje eufemístico, bajo el argumento de que estos reactivos no eran comprensibles a los niños. Al final, reportaron una escala unidimensional con una varianza explicada de $5 \%$ y una confiabilidad de .85 .

Haro (2014) adaptó el instrumento a partir de una muestra de 160 estudiantes de secundarias del centro de México. Por medio de análisis factorial por componentes principales y rotación varimax, encontró dos dimensiones: (a) justificación moral y (b) distorsión de lenguaje y comparación ventajosa, lo que es contrario a lo reportado por Obermann (2011b) y Pelton et al., (2004). A su vez, cada dimensión contiene cuatro reactivos; la primera explicó $19.35 \%$ de la varianza, mientras que la segunda explicó 19.18\%. La confiabilidad total a través del valor de Alpha de Cronbach fue de 0.73.

Sin embargo, a pesar de los esfuerzos por validar el instrumento, se denotan inconsistencias en la estructura interna del constructo, dado que en algunas ocasiones se presenta como unidimensional (Obermann, 2011b; Pelton et al., 2004), en otras como 
bidimensional (Haro, 2014), y ninguno con las ocho dimensiones que teóricamente propusieron Bandura et al. (1996). En el caso de México, es pertinente mencionar que solo se identificaron dos estudios desarrollados de validación psicométrica: uno fue con estudiantes de un ecosistema del centro de México (Haro, 2014) y el otro validó un instrumento para medir la desconexión moral en estudiantes de primaria (García, Valdés, Carlos, y Alcántar, 2019).

En función de lo expuesto, se reconoce el valor del constructo mecanismos de desconexión moral para el estudio y comprensión de conductas que no se ajustan a los estándares morales de la sociedad, como sería el caso del bullying. A su vez, se reconoce la importancia de ajustar el instrumento desarrollado para adaptarlo a las particularidades de la población de una región o país. Asimismo, se considera que la validación de los instrumentos psicológicos depende de la cultura donde hayan sido elaborados (Reyes-Lagunes, 1993) y de los análisis estadísticos utilizados para dicho propósito (Fabrigar, Wegener, MacCallum, y Strahan, 1999), es por ello que el presente estudio tiene como objetivo traducir, adaptar y evaluar las evidencias de validez por constructo y concurrente del instrumento que mide los mecanismos de desconexión moral en adolescentes mexicanos escolarizados. La evidencia de validez concurrente se estudió con los puntajes de la medida del rol de alentadores de la violencia, bajo la hipótesis de que los mecanismos de desconexión moral se relacionan de manera positiva con este rol (Haro, 2014; Obermann, 2011a; Thornberg y Jungert, 2013).

\section{MÉTODO}

\section{Participantes}

Basados en una perspectiva metodológica cuantitativa, un alcance descriptivo instrumental (Ato, López, y Benavente, 2013) y mediante un muestreo no probabilístico, por cuotas se seleccionaron a 1,212 estudiantes de 10 secundarias públicas, técnicas y generales de cinco municipios de un estado del noroeste de México. El $54.5 \%$ son mujeres y $45.5 \%$ son hombres. La media de edad de las mujeres es de 13.11 (DT=.99), mientras que la de los hombres es de 13.10 (DT=13.10). El 41\% cursa el primer grado; $29 \%$ y $30 \%$ el segundo y tercer grado, respectivamente. Por último, el $30 \%$ de los participantes informaron que además de estudiar desempañan actividades laborales. La muestra fue seleccionada de manera no probabilística por cuotas, debido a que la selección de los estudiantes se fijó a priori y solo se contempló a estudiantes que viven en polígonos con altos reportes de violencia intrafamiliar, lo cual se plantea como un vacío de investigación, también es una limitante debido a la amplia diversidad étnica y cultural no solo de la región seleccionada, sino de México (García et al., 2019). 


\section{Instrumentos}

Mecanismos de desconexión moral

Se utilizó el instrumento de Bandura et al. (1996), en el cual se proponen ocho mecanismos de desconexión moral representado por un subconjunto de cuatro reactivos, los cuales siguen una escala de respuesta tipo Likert de tres puntos indicando el grado de acuerdo o desacuerdo con los reactivos. Siguiendo la versión de Obermann (2011b), cuyo instrumento tiene los siguientes indicadores de ajuste para la validez de constructo en la versión para adolescentes daneses: $X^{2}(464)=1,671.72, P=.000, R M S E A=0.067$, 90\% CI [.064-.070], $C F I=.85, S R M R=.88$; en esta investigación, se utilizó la escala de respuesta tipo Likert, el continuo de respuesta se amplió a cinco puntos, los cuales van de 1 (totalmente en desacuerdo) a 5 (totalmente de acuerdo) y consta de 32 ítems.

\section{Escala de rol de espectadores}

Para evidenciar la validez concurrente se midió el rol de observadores alentadores de la agresión en situaciones de agresión entre pares, utilizando la subescala de Belacchi y Farina (2010) adaptada por González, Vera, Peña, y Durazo (2020) con una población de estudiantes de secundaria de la región noroeste de México, la cual presentó para la escala total: $C F I=.96, G F I=.96, R M S E A=.050$, y Alpha de Cronbach $=.82$, lo que indica propiedades psicométricas adecuadas. La escala mide distintas conductas de los estudiantes vinculados al rol de observadores de una situación hipotética de acoso: "Reforzador" (pro agresor), "Defensor" (pro víctima) y "Evasivo" (no participante). En la adaptación del instrumento quedaron 13 ítems cerrados en una escala tipo Likert de cinco puntos: (1) Nunca, (2) Casi nunca, (3) A veces, (4) Casi siempre, y (5) Siempre.

\section{Procedimiento}

Primero se llevó a cabo una adaptación de la medida en inglés por el método de traducción-re-traducción de la escala de desconexión moral. A continuación se realizó la validez de contenido, un procedimiento necesario cuando se utiliza "un instrumento que fue construido para una población diferente, pero que se adaptó mediante un procedimiento de traducción [para alcanzar una] equivalencia semántica" (Escobar-Pérez y Cuervo-Martínez, 2008, p. 27).

Posteriormente se capacitaron 10 psicólogos en los estándares de aplicación y consideraciones éticas a seguir en el trabajo de campo y se usaron hojas electrónicas para consignar las respuestas de los estudiantes. La aplicación se realizó durante el horario de clases de manera grupal en una sola sesión con una duración aproximada de 40 minutos.

Dicha aplicación se alineó a los estándares éticos vigentes contando con el consentimiento informado de directores, docentes, padres y de los mismos estudiantes participantes (Artículos 118 y 122 del Código ético del psicólogo de la Sociedad 
Mexicana de Psicología, 2007). Asimismo, la investigación también se basó en el artículo 136 de dicho código ético, que hace referencia a la confidencialidad, ya que se informó acerca de la utilización de los datos recopilados, así como el uso anónimo de los mismos.

Una vez obtenidos los datos, el procesamiento se realizó de manera electrónica en un lector óptico "scantron", el cual genera una salida de datos con formato Excel. Posteriormente, se consignaron en una única base de datos en SPSS para Windows V20. Luego se realizó un análisis de frecuencia para ubicar valores perdidos por reactivos y por casos.

En lo que corresponde con el análisis de datos, primero se realizaron análisis descriptivos y de dispersión de los ítems de los dos instrumentos. Enseguida, se concretó un análisis de Rasch para valorar la uni-dimensionalidad de cada factor a través de los indicadores de índice de consistencia interna y externa, la correlación biserial, la dificultad y discriminación de los reactivos (González, 2008), dado que este procedimiento permite establecer propiedades invariantes entre poblaciones (Attorresi, Lozzia, Abal, Galibert, y Aguerri, 2009); y tercero, un análisis confirmatorio para observar el ajuste de los reactivos a un constructo teórico (Cea, 2002) a través del método de estimación de máxima verosimilitud: para evaluar el ajuste del modelo se utilizaron los índices ji-cuadrado $(\chi 2)$, la razón entre $\chi 2$ y el número de grados de libertad $(\chi 2 / \mathrm{gl})$, raíz del residuo cuadrático promedio estandarizado (SRMR) y raíz del residuo cuadrático promedio de aproximación con su intervalo de confianza (RMSEA IC 90), índice de bondad de ajuste comparativo (CFI) y el índice de parsimonia con el criterio de información de Akaike (AIC), donde se consideraron como aceptables los valores de $\mathrm{X}^{2}$ con $\mathrm{p}>.001 ; \mathrm{CFI} \geq .92 ; \mathrm{y}$ SRMR $\leq .08$ y RMSEA $\leq .07$. cuando CFI es mayor que .92 y AIC cercanos a cero, cuando la muestra es mayor de 250 personas y entre 12 y 30 variables (Hair, Anderson, Tatham, y Black, 2014).

Para evitar que las estimaciones se encuentren sesgadas, se utilizó un re muestreo con boostrap con 500 repeticiones utilizando la técnica de percentiles con un intervalo de confianza del $95 \%$, donde los parámetros estimados no pasaron por cero y no fueron de gran tamaño. Al final, los datos se analizaron mediante el paquete estadístico Winstep, SPSS 20, AMOS y JASP para comprobar la fiabilidad.

\section{RESULTADOS}

En primer lugar, se realizó la validación de facie o aparente del instrumento (Susana y Urbina, 1998), en la cual participaron 20 estudiantes para revisar: (a) claridad y congruencia del formato del instrumento; (b) claridad y con un nivel de dificultad apropiado de los reactivos para los escolares de secundaria; (c) el deletreo de los términos difíciles y si el espaciado de líneas eran los apropiados; (d) claridad, suficiencia 
y adecuación del instrumento; y (e) calidad de impresión de la guía de reactivos (Oluwatayo, 2012). De acuerdo con este proceso se modificaron algunos reactivos en su sintaxis y se ajustaron algunos sustantivos con el fin de que fueran más accesibles para los jóvenes de 11 a 15 años.

Tabla 1. Análisis descriptivo de los reactivos de los mecanismos de desconexión moral

\begin{tabular}{|c|c|c|c|c|}
\hline Reactivos & $\Sigma$ & 点 & D & $\frac{n}{0}$ \\
\hline JM: Está bien pelear para proteger a mis amigos. & 1.63 & 1.40 & .30 & -1.16 \\
\hline LE: Golpear a un compañero es solamente una forma de jugar. & .99 & 1.26 & 1.07 & -.02 \\
\hline $\begin{array}{l}\text { CV: Dañar una propiedad ajena no es tan malo si se compara con golpear a los } \\
\text { compañeros. }\end{array}$ & 1.08 & 1.35 & .99 & -.33 \\
\hline $\begin{array}{l}\text { DR: Los jóvenes pandilleros no deben ser culpados por los problemas que su pandilla } \\
\text { realiza. }\end{array}$ & 1.51 & 1.47 & .49 & -1.14 \\
\hline $\begin{array}{l}\text { DRP: Si un joven vive en condiciones desfavorables no se le puede juzgar por } \\
\text { comportarse agresivamente. }\end{array}$ & 1.54 & 1.40 & .46 & -1.03 \\
\hline DC: Está bien decir pequeñas mentiras porque no hacen ningún daño. & 1.29 & 1.33 & .70 & -.66 \\
\hline DH: Algunas personas merecen ser tratados como objetos. & .62 & 1.16 & 1.84 & 2.22 \\
\hline AC: Si un estudiante pelea y se porta mal en la escuela, la culpa es del maestro. & .80 & 1.19 & 1.33 & .63 \\
\hline JM: Está bien golpear a alguien que ofende a mi familia. & 1.43 & 1.40 & .55 & -.97 \\
\hline LE: Está bien golpear a un compañero odioso para que aprenda a respetar. & 1.16 & 1.33 & .86 & -.45 \\
\hline CV: Robar poco dinero no es tan malo comparado con aquellos que roban mucho. & .80 & 1.27 & 1.42 & .71 \\
\hline DR: Un joven que motiva a romper las reglas no debe ser culpado si otros le hacen caso. & 1.13 & 1.35 & .94 & -.39 \\
\hline $\begin{array}{l}\text { DRP: Si los jóvenes son indisciplinados no deberían ser culpados por su mal } \\
\text { comportamiento. }\end{array}$ & 1.22 & 1.39 & .83 & -.62 \\
\hline $\begin{array}{l}\text { DC: A los jóvenes no les molesta ser objeto de burlas porque esto demuestra que los } \\
\text { demás se interesan en ellos. }\end{array}$ & 1.15 & 1.36 & .86 & -.54 \\
\hline DH: Está bien tratar mal a alguien que se comporta como cretino. & 1.22 & 1.34 & .80 & -.59 \\
\hline $\begin{array}{l}\text { AC: Si la gente no se preocupa en donde deja sus pertenencias es su culpa que se las } \\
\text { roben. }\end{array}$ & 1.77 & 1.50 & .24 & -1.33 \\
\hline JM: Está bien pelear cuando el honor del grupo está bajo amenaza. & 1.16 & 1.37 & .90 & -.46 \\
\hline LE: Agarrar la bicicleta de alguien sin su permiso solo es agarrarla prestada. & .84 & 1.24 & 1.33 & .57 \\
\hline CV: Está bien insultar a un compañero de clase porque golpearlo es peor. & 1.04 & 1.30 & 1.01 & -.19 \\
\hline DR: Si un grupo de niños hacen algo dañino, sería injusto culpar a cualquiera de ellos. & 1.22 & 1.42 & .82 & -.71 \\
\hline $\begin{array}{l}\text { DRP: Los alumnos no pueden ser culpados por usar palabras ofensivas cuando todos sus } \\
\text { amigos lo hacen. }\end{array}$ & 1.26 & 1.26 & .75 & -.67 \\
\hline DC: Burlarse de alguien en realidad no le hace daño. & 1.01 & 1.35 & 1.06 & -.21 \\
\hline DH: Alguien que es odioso no merece ser tratado como un ser humano. & 1.10 & 1.35 & .96 & -.35 \\
\hline AC: Los compañeros que son maltratados usualmente hacen cosas para merecerlo. & 1.27 & 1.33 & .72 & -.64 \\
\hline JM: Está bien mentir para evitarle problemas a tus amigos. & 1.16 & 1.28 & .80 & -.49 \\
\hline LE: No es algo malo drogarse de vez en cuando. & .86 & 1.37 & 1.30 & .31 \\
\hline $\begin{array}{l}\text { CV: Comparado con lo que otros roban, tomar algo de una tienda sin pagar no es tan } \\
\text { malo. }\end{array}$ & .83 & 1.34 & 1.44 & .64 \\
\hline DR: Es injusto culpar a un niño que participó poco en el daño causado por su grupo. & 1.23 & 1.35 & .80 & -.58 \\
\hline $\begin{array}{l}\text { DRP: Los alumnos no son culpables por su mal comportamiento si son presionados para } \\
\text { actuar así. }\end{array}$ & 1.40 & 1.37 & .57 & -.91 \\
\hline DC: Los insultos entre compañeros no hacen daño a nadie. & 1.16 & 1.37 & .87 & -.54 \\
\hline DH: Algunas personas deben ser maltratados porque no tienen sentimientos. & .85 & 1.27 & 1.29 & .37 \\
\hline $\begin{array}{l}\text { AC: Los jóvenes no son culpables por su mal comportamiento si sus padres los presionan } \\
\text { mucho. }\end{array}$ & 1.40 & 1.40 & .63 & -.88 \\
\hline
\end{tabular}

Por ejemplo, "Abofetear y empujar a un compañero es solamente una forma de jugar" se cambió por "Golpear a un compañero es solamente una forma de jugar", y "Algunas personas merecen ser tratadas como animales" por "Algunas personas merecen ser tratadas como objetos". 
A continuación, se presentan los análisis descriptivos de los reactivos, tanto de la escala de desconexión moral como de la escala de observadores alentadores. En la tabla 1 se presentan las medias de los reactivos, se puede interpretar como que la mayoría de los estudiantes están más bien en desacuerdo con las afirmaciones del instrumento. Además, los valores de sesgo y curtosis sugieren que la distribución de las respuestas en los reactivos tiende a la normalidad univariada.

En la tabla 2 se presentan la medias y desviación típica de los reactivos relacionados con la subescala de observadores alentadores, lo cual se puede interpretar que la mayoría de los estudiantes están más bien en desacuerdo con las afirmaciones del instrumento. Además, los valores de sesgo y curtosis sugieren que la distribución de las respuestas en los reactivos tiende a la normalidad univariada.

Tabla 2. Análisis descriptivo de los reactivos de la subescala de observadores alentadores

\begin{tabular}{|c|c|c|c|c|}
\hline & $\Sigma$ & 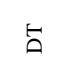 & $\begin{array}{l}8 \\
\text { s. } \\
\text { s } \\
n\end{array}$ & 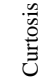 \\
\hline Me divierto en estas situaciones & 0.95 & 1.13 & 1.00 & 0.16 \\
\hline Aliento al agresor/a a continuar con su conducta & 0.55 & 0.95 & 1.81 & 2.74 \\
\hline Invito a otros compañeros/as a presenciar la situación & 0.58 & 0.95 & 1.65 & 2.16 \\
\hline Ayudo al agresor/a agarrando o sujetando a la víctima & 0.37 & 0.88 & 2.51 & 5.76 \\
\hline
\end{tabular}

Como parte del proceso de validez de constructo se realizó primero un análisis de las propiedades de los ítems utilizando el modelo de crédito parcial de la familia de la teoría Rasch, donde se encontró que todos los reactivos del instrumento de mecanismos de desconexión moral cumplieron con la normatividad de Infit y Outfit, punto biserial y discriminación empírica, pues sus rangos se encontraron entre los límites aceptados por el modelo (González, 2008), por lo que se puede concluir que todos los reactivos forman parte de un constructo denominado desconexión moral.

Posteriormente, se realizó un análisis factorial confirmatorio respetando la estructura factorial original (Bandura et al., 1996) y estos fueron los indicadores de ajuste: $X^{2}=48.53 ; g l=20 ; p=.000 ; X^{2} / g l=2.427 ; C F I=0.98 ; R M S E A=0.06$ IC 90 [0.039-0.081]; SRMR=0.03; AIC $=80.53$, los cuales se pueden considerar aceptables. No obstante, los valores de covarianza estuvieron por arriba de .80 y la revisión de los índices de modificación sugerían altas covarianzas entre los errores de los reactivos.

A partir de lo anterior, se sumaron los reactivos por dimensiones para incluir en un solo modelo la desconexión moral y la conducta de observadores alentadores de la agresión para establecer evidencia de validez concurrente y se obtuvieron indicadores aceptables: $X^{2}=109.078 ; g l=53 ; p=.000 ; X^{2} / g l=2.03 ; C F I=0.97 ; R M S E A=0.05 I C 90$ [0.038-0.065]; SRMR=0.03; AIC=159.07.

Además, el valor de beta estandarizado, que se obtuvo en el modelo de análisis concurrente $(\beta=0.53 ; p=0,001)$, permite sostener que existe una relación entre 
los mecanismos de desconexión moral y el actuar como observadores que alientan la agresión. Lo que nos indica, además, una relación positiva entre las dos variables, asumiendo, que al aumento de una, la otra lo hará de manera directa y proporcional.

Los reactivos asociados a cada una de las dimensiones tienen pesos de regresión que nos permiten indicar que todos ellos son igualmente importantes, dados sus valores encontrados y la variación entre ellos (ver Figura 1). Y, finalmente, los valores del coeficiente $\Omega$ de McDonald, que indica la fiabilidad de las escalas, en las dos medidas tienen valores por encima de .65 (.93 para la desconexión moral y .67 para la sub escala para medir el observador alentador) lo que sugiere una adecuada y buena fiabilidad (Katz, 2006).

Figura 1. Modelo de validez concurrente de la escala de Desconexión moral con el ser alentador de la agresión

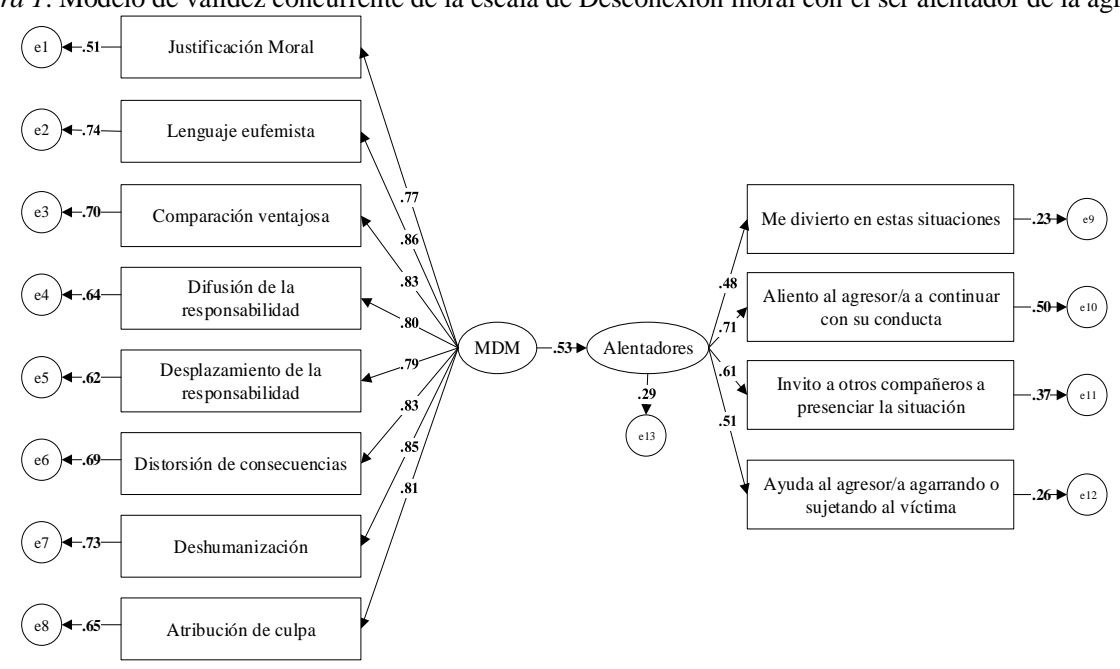

\section{DICUSIÓN Y CONCLUSIONES}

El presente estudio tiene como objetivo traducir, adaptar y evaluar las evidencias de validez por constructo y concurrente del instrumento que mide los mecanismos de desconexión moral en adolescentes mexicanos escolarizados. Tal como se esperaba, los resultados fueron similares a los encontrados en otros ecosistemas y culturas, (Haro, 2014; Pelton et al., 2004). Es decir, el instrumento presente se ajusta al modelo de ocho factores, pero presenta altos valores en las covarianzas, lo cual sugiere que los reactivos están altamente relacionados y todos permiten medir el constructo denominado desconexión moral (Bandura et al., 1996).

En lo que respecta a la hipótesis de que a mayor acuerdo con los mecanismos de desconexión moral, mayor la probabilidad de asumir el rol de observador alentador en 
tales situaciones, se encontró que los resultados son similares a lo encontrado en otros estudios (Barchia y Bussey, 2011; Doramajian y Bukowski 2015; Gini, 2006; Gini, Pozzoli y Hauser, 2011; Menesini et al., 2003; Obermann, 2011a, 2011b). Lo anterior nos permite comprobar que los mecanismos de desconexión propuestos inicialmente por Bandura (2002) para la conducta agresiva, se cumplen en el bullying.

Se recomienda en próximas investigaciones reflexionar sobre la parsimonia de la escala, sobre todo considerando que todas las dimensiones tienen altas correlaciones, sobre todo si el instrumento se pretende utilizar en investigaciones que requieran análisis multivariados. Una manera de hacerlo es enfatizar en algunos problemas que se contraponen con los estándares morales de los estudiantes, pues como se ha demostrado, a veces a los adolescentes les cuesta diferenciar las conductas por su edad (Pelton et al., 2004).

La validación de este instrumento aporta insumos para realizar análisis contextualizados, producto del uso de instrumentos con parámetros psicométricos adecuados que permiten dar continuidad al estudio de este fenómeno del acoso escolar y las variables que lo conforman, que lamentablemente continúan afectando de manera global a las instituciones educativas, convirtiéndose en una preocupación compartida, dados sus incontables efectos sobre la población escolar.

La continuidad e incremento de la prevalencia del bullying han obligado a los investigadores e interesados en el tema a indagar de manera más exhaustiva todas las posibles variables, mecanismos y procesos (individuales, grupales y sociales) que contribuyen a su mantenimiento, datos en los que se han evidenciado mecanismos más finos, como el de la desconexión moral, que ameritan ser reconocidos y evaluados en sus dimensiones y características. Asimismo, a identificar las variables que contribuyan a reducir la incidencia de la agresión entre pares y a atenuar sus efectos (Cándido et al., 2014; Nacimiento y Mora, 2014).

Los escasos estudios longitudinales existentes han mostrado la estabilidad de los mecanismos de desconexión moral y su tendencia a hacerse más rígidos en cuanto se tiene mayor edad, así como su mayor prevalencia entre los hombres. Verificar estos hallazgos en poblaciones diferentes a las investigadas es viable cuando se cuenta con instrumentos adecuados que estén validados en los contextos en que se utilicen, de manera tal que permitan su detección y caracterización. Esto, a su vez, redundará en poder incluir dichos mecanismos de desconexión moral, detectados y medidos, en toda acción o programa que tenga como propósito prevenir y reducir el acoso escolar, fortaleciendo el compromiso moral de los estudiantes en la prevención y la detención del fenómeno, mecanismo inverso a la desconexión moral. 


\section{REFERENCIAS}

Ato, M., López, J., y Benavente, A. (2013). Un sistema de clasificación de los diseños de investigación en psicología. Anales de psicología, 29(3), 1038-1059. doi:10.6018/analesps.29.3.178 511

Attorresi, F., Lozzia, S., Abal, J., Galibert, S., y Aguerri, E. (2009). Teoría de Respuesta al Ítem. Conceptos básicos y aplicaciones para la medición de constructos psicológicos. Revista Argentina de Clínica Psicológica, 28(2),179-188.

Bandura, A. (1975). Análisis del aprendizaje social de la agresión y la delincuencia. En A. Baudura y E. Ribes (Eds.), Modificación de Conducta. Análisis de la agresión y la delincuencia (pp. 307-350). Ciudad de México: Trillas.

Bandura, A. (1990). Mechanisms of moral disengagement. En W. Reich (Ed.), Origins of terrorism: psychologies, ideologies, states of mind (pp. 161-191). Washington, D.C: Woodrow Wilson Center Press.

Bandura, A. (2002). Selective moral disengagement in the exercise of moral agency. Journal of Moral Education, 31(2), 101-119. doi:10.1080/0305724022014322

Bandura, A. (2016). Moral disengagement: How people do harm and live with themselves. Moral disengagement: How people do harm and live with themselves (1era ed.). New York: Worth.

Bandura, A., Barbaranelli, C., Caprara, G.V., y Pastorelli, C. (1996). Mechanisms of moral disengagement in the exercise of moral agency. Journal of Personality and Social Psychology, 71(2), 364-374. doi:10.1037/0022-3514.71.2.364

Bandura, A., Caprara, G., Barbaranelli, C., Pastorelli, C., y Regalia, C. (2001). Sociocognitive self-regulartory mechanisms governing transgressive behavior. Journal of Personality and Social Psychology, 80(1), 125-135. doi:10.1037/0022-3514.80.1.125

Barchia, K., y Bussey, K. (2011). Predictors of student defenders of peer aggression victims: Empathy and social cognitive factors. International Journal of Behavioral Development, 35, 289-297. doi:10.1177/0165025410396746

Belacchi, C., y Farina, E. (2010). Prosocial/hostile roles and emotion comprehension in preschoolers. Aggressive Behavior, 36(6), 371-389. doi:10.1002/ab.20361

Bjärehed, M., Thornberg, R., Wänström, L., y Gini, G. (2019). Mechanisms of moral disengagement and their associations with indirect bullying, direct bullying, and proaggressive bystander behavior. Journal of Early Adolescence, 40(1), 28-55. doi: $10.1177 / 0272431618824745$

Campaert, K., Nocentini, A., y Menesini, E. (2017). The efficacy of teachers' responses to incidents of bullying and victimization: The mediational role of moral disengagement for bullying. Aggressive Behavior, 43(5), 483-492. doi:10.1002/ab.21706

Cándido, I., Torregosa, M., García, J., Martínez., M., Estévez, E., y Delgado, B. (2014). Conducta agresiva e inteligencia emocional en la adolescencia. European Journal of Education and Psychology, 7(1), 29-41. doi:10.1989/ejep.v7i1.150

Caravitaa, S., Strohmeierb, D., Salmivalli, C., y Di Blasioa, P. (2019). Bullying immigrant versus non-immigrant peers: Moral disengagement and participant roles. Journal of School Psychology, 75, 119-133. doi:10.1016/j.jsp.2019.07.005

Cea, M. Á. (2002). Análisis multivariable: teoría y práctica en la investigación social. España: Síntesis.

Comisión Económica para América Latina y el Caribe. (2017). Las violencias en el espacio escolar. Santiago: ONU.

Comisión Económica para América Latina y el Caribe. (2016). Horizons 2030: Equality at the Centre of Sustainable Development. Santiago: ONU. 
Cuevas, M. y Marmolejo, M. (2016). Observadores: un rol determinante en el acoso escolar. Pensamiento Psicológico, 14(1), 89-102. doi:10.11144/Javerianacali.PPSI14-1.orda

Doramajian, C., y Bukowski, W.M. (2015). A longitudinal study of the associations between moral disengagement and active defending versus passive bystanding during bullying situations. Merrill-Palmer Quarterly, 61(1), 144-172.

Escobar-Pérez, J., y Cuervo-Martínez, Á. (2008). Validez de contenido y juicio de expertos: una aproximación a su utilización. Avances en Medición, 6, 27-36.

Fabrigar, L.R., Wegener, D.T., MacCallum, R.C., y Strahan, E.J. (1999). Evaluating the use of exploratory factor analysis in psychological research. Psychological Methods, 4(3), 272-299. doi:10.1037/1082-989X.4.3.272

Garaigordobil, M., Mollo-Torrico, J. P., y Larrain, E., (2018). Prevalencia de bullying y cyberbullying en Latinoamérica: una revisión. Revista Iberoamericana de Psicología, $11(3), 1-18$.

García, F., Valdés, A., Carlos, E., y Alcántar, C. (2019). Propiedades psicométricas de una escala para medir desconexión moral en niños mexicanos. Acta Colombiana de Psicología, 22, 118-128. doi:10.14718/ACP.2019.22.1.6

Gini, G. (2006). Social cognition in bullying: What's wrong? Aggressive Behavior, 32, 528-539. doi:10.1002/ab.21574

Gini, G., Pozzoli, T., y Hauser, M. (2011). Bullies have enhanced moral competence to judge relative to victims, but lack moral compassion. Personality and Individual Differences, 50, 603-608. doi:10.1016/j.paid.2010.12.002

Gini, G., Thornberg, R., y Pozzoli, T. (2020). Individual moral disengagement and bystander behavior in bullying: The role of moral distress and collective moral disengagement. Psychology of Violence, 10(1), 38-47. doi:10.1037/vio0000223

González, E., Vera, J., Peña, M. y Durazo, F. (2020). Propiedades métricas de una escala para medir roles asociados de los espectadores del acoso escolar en alumnos mexicanos de secundaria. Educación y Humanismo, 22(38), 1-12. doi: 10.17081/eduhum.22.38.3611

González, M. (2008). El Análisis de Reactivos con el Modelo Rasch Manual Técnico A Serie: Medición y Metodología. Recuperado de: http://www.winsteps.com/a/recursosoffline.pdf

Hair, J., Anderson, R., Tatham, R., y Black, W. (2014). Multivariate Data Analysis (7ta ed.). United Kingdom: Pearson.

Haro, I. (2014). Factores vinculados con el papel que asumen los alumnos involucrados en el maltrato entre iguales (bullying). Ciudad de México: Universidad Nacional Autónoma de México.

Herrera-López, M., Romera, E., y Ortega-Ruiz, R. (2018). Bullying y Cyberbullying en Latinoamérica. Un estudio bibliométrico. Revista Mexicana de Investigación Educativa, 23(76), 125-155.

Katz, M. (2006). Multivariable analysis. Cambridge: Cambridge University Press.

Lopez, M., y Kyriacou, C. (2016). Self-efficacy and moral disengagement in Mexican secondary school bullying bystanders. Psychology of Education Review, 40(2), 28-32.

Menesini, E., Sánchez, V., Fonzi, A., Ortega, R., Costable, A., y Lo Feudo, G. (2003). Moral emotions and bullying: A cross-national comparison of differences between bullies, victims and outsiders. Aggressive Behavior, 29, 515-530. doi:10.1002/ab.10060

Meter, D., y Bauman, S. (2018). Moral Disengagement About Cyberbullying and Parental Monitoring: Effects on Traditional Bullying and Victimization via Cyberbullying Involvement. Journal of Early Adolescence, 38(3), 303-326. doi:10.1177/0272431616670752 
Nacimiento, L., y Mora, J. (2014). El uso de estrategias de afrontamiento y habilidades metacognitivas ante situaciones de bullying y cyberbullying. European Journal of Education and Psychology, 7(2), 121-129. doi:10.1989/ejep.v7i2.184

Obermann, M.L. (2011a). Moral disengagement among bystanders to school bullying. Journal of School Violence, 10(3), 239-257. doi:10.1080/15388220.2011.578276

Obermann, M.L. (2011b). Moral disengagement in self-reported and peer-nominated school bullying. Aggressive Behavior, 37(2), 133-144. doi:10.1002/ab.20378

Oluwatayo, J.A. (2012). Validity and reliability issues in educational research. Journal of Educational and Social Research, 2(2), 391-400.

Olweus, D., Solberg, M., y Breivik, K. (2020). Long-term school-level effects of the Olweus Bullying Prevention Program (OBPP). Scandinavian Journal of Psychology, 61, 108116. doi:10.1111/sjop. 12486

Ortega, R., Sánchez, V., y Menesini, E. (2002). Violencia entre iguales y desconexión moral: Un análisis transcultural. Psicothema, 14(Extra 1), 37-49.

Paulino, L.R., Avilés, J.M., y Sales, P.J. (2016). Bullying, un problema moral: representaciones de sí mismo y desconexiones morales. Revista de Educación, (373), 9-34. doi:10.4438/1988-592X-RE-2016-373-319

Pelton, J., Gound, M., Forehand, R., y Brody, G. (2004). The Moral Disengagement Scale: Extension with an american minority sample. Journal of Psychopathology and Behavioral Assessment, 26(1), 31-39. doi:10.1023/B:JOBA.0000007454.34707.a5

Reyes-Lagunes, I. (1993). Las redes semánticas naturales, su conceptualización y su uso en la construcción de instrumentos. Revista de Psicología Social y Personalidad, 9, 83-99.

Sociedad Mexicana de Psicología. (2007). Código ético del psicólogo (4ta ed.). Ciudad de México: Trillas.

Susana, A., y Urbina, S. (1998). Tests psicológicos. Ciudad de México: Prentice Hall.

Thornberg, R., y Jungert, T. (2013). Bystander behavior in bullying situations: Basic moral sensitivity, moral disengagement and defender self-efficacy. Journal of Adolescence, 36, 475-483. doi:10.1016/j.adolescence.2013.02.003

Vega-Cauich, J. (2019). Prevalencia del bullying en México: un meta-análisis del bullying tradicional y cyberbullying. Diversitas: Perspectivas en Psicología, 15(1), 113-129. doi:10.15332/22563067.4020

Wang, C., Hoon, J., Swearer, S., Turner, R. y Goldberg, T. (2017). Longitudinal relationships between bullying and moral disengagement among adolescents. Journal Youth Adolescence, 46, 1304-1317. doi:10.1007/s10964-016-0577-0

Recibido: 4 de junio de 2020

Recepción Modificaciones: 19 de junio de 2020

Aceptado: 2 de julio de 2020 
BAUTISTA et al. Instrumento desconexión moral en adolescentes

Anexo

Instrumento de desconexión moral traducido al español

\begin{tabular}{|c|c|}
\hline Factores & Reactivos \\
\hline \multirow{4}{*}{ Justificación moral } & Está bien pelear para proteger a mis amigos. \\
\hline & Está bien golpear a alguien que ofende a mi familia. \\
\hline & Está bien pelear cuando el honor del grupo está bajo amenaza. \\
\hline & Está bien mentir para evitarle problemas a tus amigos. \\
\hline \multirow{4}{*}{ Lenguaje eufemista } & Golpear a un compañero es solamente una forma de jugar. \\
\hline & Está bien golpear a un compañero odioso para que aprenda a respetar. \\
\hline & Agarrar la bicicleta de alguien sin su permiso solo es agarrarla prestada. \\
\hline & No es algo malo drogarse de vez en cuando. \\
\hline \multirow{4}{*}{ Comparación ventajosa } & $\begin{array}{l}\text { Dañar una propiedad ajena no es tan malo si se compara con golpear a los } \\
\text { compañeros. }\end{array}$ \\
\hline & Robar poco dinero no es tan malo comparado con aquellos que roban mucho. \\
\hline & Está bien insultar a un compañero de clase porque golpearlo es peor. \\
\hline & $\begin{array}{l}\begin{array}{l}\text { Comparado con lo que otros roban, tomar algo de una tienda sin pagar no es tan } \\
\text { malo. }\end{array} \\
\end{array}$ \\
\hline \multirow{4}{*}{$\begin{array}{l}\text { Difusión de la } \\
\text { responsabilidad }\end{array}$} & $\begin{array}{l}\begin{array}{l}\text { Los jóvenes pandilleros no deben ser culpados por los problemas que su pandilla } \\
\text { realiza. }\end{array} \\
\end{array}$ \\
\hline & Un niño que motiva a romper las reglas no debe ser culpado si otros le hacen caso. \\
\hline & Si un grupo de niños hacen algo dañino, sería injusto culpar a cualquiera de ellos. \\
\hline & Es injusto culpar a un niño que participo poco en el daño causado por su grupo. \\
\hline \multirow{4}{*}{$\begin{array}{l}\text { Desplazamiento de la } \\
\text { responsabilidad }\end{array}$} & $\begin{array}{l}\text { Si un niño vive en condiciones desfavorables no se le puede juzgar por } \\
\text { comportarse agresivamente. }\end{array}$ \\
\hline & $\begin{array}{l}\text { Si los niños son indisciplinados no deberían ser culpados por su mal } \\
\text { comportamiento. }\end{array}$ \\
\hline & $\begin{array}{l}\text { Los niños no pueden ser culpados por usar palabras ofensivas cuando todos sus } \\
\text { amigos lo hacen. }\end{array}$ \\
\hline & $\begin{array}{l}\text { Los niños no son culpables por su mal comportamiento si son presionados para } \\
\text { actuar así. }\end{array}$ \\
\hline \multirow{4}{*}{$\begin{array}{l}\text { Distorsión de } \\
\text { consecuencias }\end{array}$} & Está bien decir pequeñas mentiras porque no hacen ningún daño. \\
\hline & $\begin{array}{l}\text { A los niños no les molesta ser objeto de burlas porque esto demuestra que los } \\
\text { demás se interesan en ellos. }\end{array}$ \\
\hline & Burlarse de alguien en realidad no le hace daño. \\
\hline & Los insultos entre niños no hacen daño a nadie. \\
\hline \multirow{4}{*}{ Deshumanización } & Algunas personas merecen ser tratados como objetos. \\
\hline & Está bien tratar mal a alguien que se comporta como cretino. \\
\hline & Alguien que es odioso no merece ser tratado como un ser humano. \\
\hline & Algunas personas deben ser maltratados porque no tienen sentimientos. \\
\hline \multirow{4}{*}{ Atribución de culpa } & Si un estudiante pelea y se porta mal en la escuela la culpa es del maestro. \\
\hline & $\begin{array}{l}\begin{array}{l}\text { Si la gente no se preocupa en donde deja sus pertenencias es su culpa que se las } \\
\text { roben. }\end{array} \\
\end{array}$ \\
\hline & Los niños que son maltratados usualmente hacen cosas para merecerlo. \\
\hline & $\begin{array}{l}\begin{array}{l}\text { Los niños no son culpables por su mal comportamiento si sus padres los presionan } \\
\text { mucho. }\end{array} \\
\end{array}$ \\
\hline
\end{tabular}

Nota. Traducida y adaptada de Bandura et al. (1996). 